\title{
Le gène de l'ataxie télangiectasie code pour une protéine ressemblant à la Pl-3 kinase
}

L'ataxie télangiectasie est une maladie héréditaire autosomique récessive frappant 1 personne sur 40 à 100000 . Elle associe un syndrome de déficience immunitaire, une sensibilité importante aux radiations ionisantes, au cancer, une dégénérescence cérébelleuse progressive et des dilatations des vaisseaux sanguins (télangiectasies) au niveau des conjonctives des yeux, et parfois de la peau du visage. Les malades meurent habituellement avant 30 ans de leur déficit immunitaire, du développement de lymphomes et d'un phénomène de vieillissement accéléré. Les cellules en culture dérivées de malades atteints d'ataxie télangiectasie ont une radiosensibilité anormale; des expériences de complémentation par formation d'hétérocaryons avaient permis de suggérer qu'existaient quatre groupes de complémentation (a, c, d, e), c'est-à-dire quatre loci différents [1]. Des études familiales avaient permis de localiser la mutation du groupe a dans la région q22-23 du chromosome 11 . Ultérieurement, le groupe $\mathrm{C}$ avait été également localisé dans cet intervalle. C'est à partir de ces données qu'un consortium international s'est lancé dans le clonage du gène, mêlant la coopération entre les groupes et, surtout à la phase finale, une compétition farouche. C'est que le gène de l'ataxie-télangiectasie pourrait avoir une importance dépassant de loin la maladie héréditaire relativement rare dont il est responsable. En effet, des études épidémiologiques ont montré la particulière fréquence de cancers du sein chez les femmes de familles dans lesquelles des cas d'ataxie télangiectasie avaient été observées. Ces résultats ont per- mis de faire l'hypothèse selon laquelle le gène de l'ataxie télangiectasie pourrait être, à l'état hétérozygote, l'un des facteurs génétiques prédominants de susceptibilité au cancer du sein et peut-être à à d'autres types de cancers [2]. Le clonage et la caractérisation du gène dénommé $A T M$ (ataxia telangiectasia mutated) ont été avant tout réalisés par l'équipe de Yosef Shiloh, de l'université de TelAviv en Israël; les résultats sont publiés dans un article du 23 juin 1995 de Science signé par vingt-trois auteurs [3]. La méthode utilisée pour isoler le gène est celle du clonage positionnel. Un contig de YAC couvrant l'intervalle de trois mégabases où avait été localisé le locus a été établi ; ces différents chromosomes artificiels de levure ont ensuite été utilisés pour capturer des ADNc hybridant à des exons contenus dans ces YAC. L'un de ces ADNc, partiel, a été trouvé modifié chez quatorze malades atteints d'ataxie télangiectasie appartenant à l'un quelconque des groupes de complémentation a, c, d, e. Les mutations observées sont des délétions, en phase ou avec décalage de phase de lecture et aboutissant alors à une protéine tronquée. Le messager ATM est présent dans tous les tissus de l'organisme et sa forme majoritaire a une taille de 12 kb. Une phase ouverte de lecture partielle de 5124 nucléotides a été séquencée, ce qui a permis de déduire la séquence en acides aminés de la partie carboxy-terminale de la protéine ATM. L'élément remarquable de cette séquence est qu'elle comporte, dans sa région la plus carboxy-terminale, un domaine de forte analogie avec la phosphatidyl-inositol 3 kinase, domaine que l'on retrouve égale- ment dans d'autres protéines de levure et de mammifères jouant un rôle dans la transmission de signaux au sein de la cellule (les protéines TOR). Une ressemblance plus faible existe avec des protéines de levure jouant un rôle dans la réparation de l'ADN et dans le contrôle du cycle cellulaire (ESRl, Rad3). La PI-3 kinase joue un rôle manifestement important dans la transmission des signaux, quoique sa régulation et les étapes situées en aval de son activation ne soient pas bien connues. Des résultats récents rappelés dans le numéro 6 de médecine/sciences $\left(\mathrm{m} / \mathrm{s} n^{\circ}\right.$ 6 , vol. 11, p. 903) indiquent que l'effet du NGF sur l'inhibition de l'apoptose de cellules chromaffines en culture passe par la PI-3 kinase. Ce résultat est particulièrement intéressant puisque l'un des symptômes de l'ataxie télangiectasie est justement l'ataxie, secondaire à une mort progressive des cellules de Purkinje. De plus, la PI-3 kinase aussi bien que les protéines TOR aboutissent à l'activation de la p70 S6 kinase $(\mathrm{m} / \mathrm{s}$ $n^{\circ} 6$, vol. 11, p. 903), et cette activation est bloquée par l'immunosuppresseur rapamycine, ce qui pourrait avoir un rapport avec le syndrome de déficience immunitaire associé à la maladie. Quant à la multiplicité des autres symptômes, elle ne peut pas étonner pour la déficience d'une protéine potentiellement impliquée dans la transmission de signaux en réponse à des agents variés. Les résultats de Savitsky et al. [2] démontrent que tous les malades testés ont une mutation du même gène $A T M$ alors qu'on les considérait initialement comme appartenant à des groupes de complémentation différents. Cela signifie que la méthode utilisée pour 
réaliser ces expériences de complémentation somatique ont abouti à des résultats erronés.

L'intérêt des résultats rapportés est considérable à plusieurs titres. D'une part, la perturbation d'un système impliqué dans la transmission des signaux comme celui des PI-3 kinases est naturellement de nature à renseigner sur le véritable rôle de ce type de protéine $\left(\left(m / s n^{\circ} 6\right.\right.$, vol. $\left.11, p .903\right)$. D'autre part, s'il se confirme qu'une mutation hétérozygote du gène $A T M$ est un facteur de susceptibilité au cancer du sein, cela pourrait avoir de très importantes conséquences pratiques. En effet, dans les familles avec une grande fréquence de cancers mammaires, la découverte d'un tel gène de prédisposition serait un très fort argument pour interdire la pratique d'examens radiologiques à répétition tels que des mammographies afin de parvenir à un dépistage précoce: la mutation du gène $A T M$ est susceptible de provoquer une très importante augmentation de la radio-sensibilité, c'est-à-dire d'augmenter le pouvoir cancérigène des radiations ionisantes. L'importance de l'enjeu de santé publique explique la vigueur de la compétition entre différentes sociétés privées pour obtenir une licence d'exploitation de ce gène, avec tous les problèmes sociaux et éthiques déjà évo- qués à l'occasion de la découverte des gènes de susceptibilité au cancer du sein BRCAl et BRCA2 $\left(\mathrm{m} / \mathrm{s} n^{\circ} 11\right.$, vol. 10, p. $1172 ; n^{\circ} 6$, vol. 11, p. 920).

A.K.

1. Aurias A. Ataxie-télangiectasie: aspects cliniques, épidémiologiques et génétiques. médecine/sciences 1994; 10: 957-61.

2. Nowak R. Discovery of AT gene sparks biomedical research Bonanza. Science 1995; 268 : 170()-1.

3. Savitsky K, Bar-Shira A, Gilad S, et al. A single ataxia telangiectasia gene with a product similar to PI-3 kinase. Science $1995 ; 268$ : 1749-53. 